\title{
Pattern and Pain Assessment of Musculoskeletal Disorders Attending to Physiotherapy Services in Selected Physiotherapy Centres of Dhaka City
}

\author{
Md Ruhul Amin'1, Sanjida Akhter², Kazi Afzalur Rahman ${ }^{3}$
}

\begin{abstract}
Background: Musculoskeletal disorders are the most common causes of severe long-term pain and physical disability, affecting hundreds of millions of people around the world. Among different modalities of treatment and management for musculoskeletal pain, physiotherapy might be cost-effective. Objective: The aim of the study was to determine the pattern and pain assessment of musculoskeletal disorders attending to physiotherapy services in selected physiotherapy centers of Dhaka city. Materials and method: It was a cross sectional study. Sample size was 400 and a pre-tested, modified, semi-structured questionnaire was used to collect the data. Data were analyzed using SPSS software version 16.0. Results: Majority of the respondents (69.2\%) belonged to 46-65 year age group with least in extreme age groups. Mean $\pm S D$ of age was $33.58 \pm 12.33$ years. Most of the respondents were male (69.2\%). Study revealed that neck pain (75.2\%) was the major complaint followed by back pain (48.00\%). Regarding pattern of pain, intermittent (73.2\%) type was predominant. Muscle spasm (29.2\%) was the main cause for musculoskeletal pain followed by degenerative diseases (27.5\%). Of the respondents $55.00 \%$ received traction, $91.25 \%$ exercise, $21.25 \%$ manipulation, 97.95\% short wave diathermy, $85.00 \%$ ultrasound therapy, $33.75 \%$ infra red ray, and $12.75 \%$ electrical stimulation as physiotherapy treatment. There were statistically significant difference between sex and severity of pain $(p=0.019)$, educational status and pain persisting time in years $(p=0.000)$. There was also statistically highly significant difference between severity of pain responses before and after physiotherapy treatment $(p=0.000)$. Conclusion: Study concluded that common areas of musculoskeletal pain were neck followed by back and shoulder and pain characteristics were intermittent, radiating, numbness, burning in nature. Respondents had taken medication and different type of physiotherapy services, including traction, exercise, short wave diathermy and ultrasound therapy.
\end{abstract}

Keywords: Musculoskeletal pain; manipulation; physiotherapy; occupational therapy.

Delta Med Col J. Jul 2015;3(2):83 - 88

\section{Introduction}

Pain is a multidimensional experience that is a prominent feature of many musculoskeletal disorders and is a highly relevant complaint. Musculoskeletal disorders are the most common causes of severe long-term pain and physical disability, affecting hundreds of millions of people around the world. In industrialized countries, which have increasingly aging populations,

1. Assistant Professor and Course coordinator, Department of Physiotherapy, SAIC Institute of Medical Technology, Dhaka, Bangladesh.

2. Assistant Professor, Department of Forensic Medicine, Delta Medical College, Dhaka, Bangladesh.

3. Assistant Professor, Department of Pharmacology, Dhaka Medical College, Dhaka, Bangladesh.

Correspondence: Md Ruhul Amin. e-mail: ruhul31physio@yahoo.com 
chronic musculoskeletal pain has become a significant health care burden. ${ }^{1}$

In a study done in United Kingdom on patients with acute and chronic musculoskeletal pain receiving physiotherapy, it was revealed that persons those in the acute group were optimistic of a good result, whereas those with chronic degenerative conditions were either doubtful of improvement or unrealistic in their hopes for complete resolution of their symptoms. ${ }^{2}$ Exercise-based intervention is an established modality of treatment in a number of medical conditions. Exercise might be cost-effective in musculoskeletal disorders, intermittent claudication, breast cancer patients, diabetes, and schizophrenia. $^{3}$ Evidence-based efficacy of different modalities of treatment for musculoskeletal pain including exercise therapy, shortwave diathermy, ultrasound therapy, transcutaneous electrical nerve stimulation is presented in different studies. ${ }^{4}$

A study was conducted in Brazil in 2002 and developed a protocol for low back pain patients by conventional physical therapy treatment, with or without home exercises programme and found that higher level of satisfaction was with home exercises group. $^{5}$ A systematic review was conducted by Shamliyan and Wang and found that short wave diathermy, orthotics, and magnetic stimulation are of no benefit in osteoarthritis knee, rather better response in exercise. ${ }^{6}$ A study conducted by Casserley-Feeney et al. found that most common site of musculoskeletal pain was spinal, male were more sufferer than female and they had previous experience of physiotherapy treatment. $^{7}$

Musculoskeletal disorders are prevalent and a major burden on individuals and society. ${ }^{8}$ Different studies showed significant association between intensity of musculoskeletal pain and reduction in work ability. ${ }^{8,9}$ Ullrich et al. demonstrated that the lower body pain was as common as upper extremity pain, but tended to be more intense. ${ }^{10}$

A study was conducted in São Paulo, Brazil and found that the proportion of reported musculoskeletal pain was $22 \%$, among which $20 \%$ was male and $27 \%$ was female and risk ratio was $1.35 .{ }^{11}$

Occupation in some cases play vital role for the occurrence of musculoskeletal disorders. A prevalence study was conducted among the Mashhad Dental students and found that $82 \%$ of undergraduate students and $90 \%$ of postgraduate students reported body pain in at least one region. The most prevalent pain locations were: chest/shoulder (46.9\%), head/neck $(41.8 \%)$, middle back (33.9\%) and right hand (25.4\%). Severity of reported pain was increased due to performing dental work. ${ }^{13}$

Anke et al. found that, pain was not associated with satisfaction with family life, partner relationship or sexual life. ${ }^{14}$ On the other hand Sustová et al. found that a statistically significant correlation with musculoskeletal complaints of medium and major intensity was demonstrated for sex and age. ${ }^{15}$ Moderate to severe pain and chronic pain had a significant impact on the occupational and social relationships as well as psychological aspect of those required multidisciplinary intervention. ${ }^{16}$

A prospective longitudinal study on older people revealed that, $48.9 \%$ chronic pain was associated with poor self-rated health, adjusted odds ratio was 2.26. ${ }^{17}$ Silva et al. reported that $77.5 \%$ respondents had chronic, $55.4 \%$ had radiating pain and $90.2 \%$ had constant pain with moderate to severe intensity. $^{18}$

A prevalence study of musculoskeletal symptoms was $38.3 \%$, was higher in females and in elderly, and had pain in the back, shoulder, and knee, in chronological order. ${ }^{19}$

\section{Materials and method}

A cross sectional study was conducted in Dhaka city to determine pattern and pain assessment of musculoskeletal disorders attending to physiotherapy services in selected physiotherapy centers with 400 subjects. The study was conducted in Metropolitan Medical Centre Limited (MMC Ltd.), Mohakhali, Dhaka, National Institute of Traumatology, and Orthopedic 
Rehabilitation (NITOR), Sher-E-Banglanagor, Dhaka, Islami Bank Central Hospital Limited (IBCH Ltd.), Dhaka and Physiotherapy department of SAIC Institute of Medical Technology, Dhaka. A pre-tested modified semi structured questionnaire was used to collect the data on the basis of objectives and variables. The data were analyzed by using statistical packages for social science (SPSS) software version 16.0 for Windows.

\section{Results}

Mean \pm SD age of the respondents were $33.58 \pm 12.33$ years with a range from 16 to 80 years.

It was found that majority of the respondents $(63.8 \%)$ belonged to age group of 36-65 years. Among the respondents, $69.2 \%$ were male and $30.8 \%$ were female. It was also found that $30.8 \%$, $33.8 \%, 18.0 \%, 8.8 \%$, and $2.5 \%$ of the respondents belonged to the level of graduate, higher secondary, post graduate, secondary and primary education respectively (Table I).

\begin{tabular}{llcc}
$\begin{array}{l}\text { Table I: } \\
\text { (N=400) }\end{array}$ & Socio-demographic & characteristics \\
\hline Variables & Categories & Frequency & Percentage \\
\hline Age (years) & $16-25$ & 6 & 1.5 \\
& $26-35$ & 42 & 10.5 \\
& $36-45$ & 80 & 20.0 \\
& $46-55$ & 132 & 33.0 \\
& $56-65$ & 123 & 30.8 \\
Sex & $>66$ & 17 & 4.2 \\
& Male & 277 & 69.2 \\
& Female & 123 & 30.8 \\
Educational & Primary & 10 & 2.5 \\
status & Secondary & 35 & 8.8 \\
& Higher & 135 & 33.8 \\
& secondary & & \\
& Graduate & 148 & 37.0 \\
& Post graduate & 72 & 18.0 \\
\hline
\end{tabular}

Table II shows that $75.2 \%, 48.00 \%, 30.8 \%, 19 \%$, $10.8 \%$, and $6 \%$ of the respondents complained about neck pain, back pain, shoulder pain, pain in upper limb, knee pain, leg and ankle pain respectively. Among the respondents $77.8 \%$ were feeling pain on over stress.
Table II: Distribution of respondents by area of musculoskeletal pain (Multiple responses)

\begin{tabular}{lcc}
\hline Area of pain & Frequency & Percentage \\
\hline Neck & 301 & 75.2 \\
Back & 123 & 48 \\
Shoulder & 76 & 30.8 \\
Upper limb & 192 & 19 \\
Knee & 43 & 10.8 \\
Leg and ankle & 24 & 6 \\
\hline
\end{tabular}

Figure 1 shows the distribution of duration of persistence of pain. It showed that most (93.5\%) of the respondents had been suffering from 1 to 5 years.

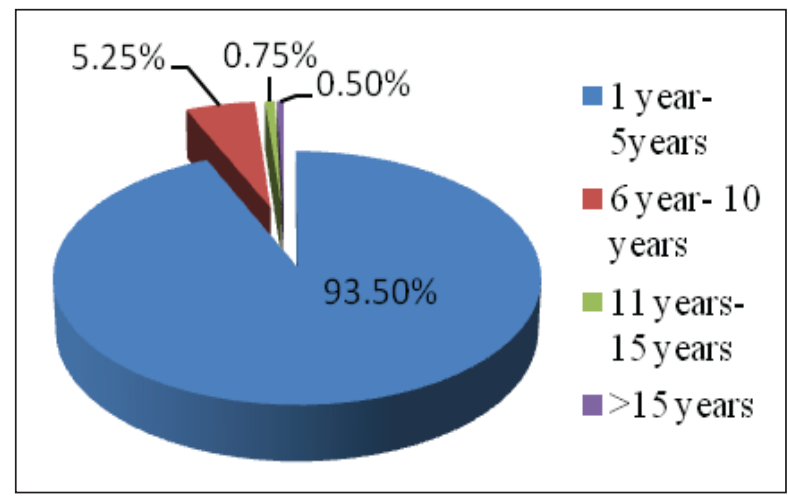

Fig 1: Distribution of respondents by duration of pain

Table III reveals that majority of the respondents (73.2\%) complained about intermittent pain followed by radiating pain and continuous pain with almost equal proportion.

Table III: Distribution of respondents by pain characteristics (Multiple responses)

\begin{tabular}{lcc}
\hline Characteristics of pain & Frequency & Percentage \\
\hline Intermittent & 293 & 73.2 \\
Continuous & 101 & 25.2 \\
Tingling & 34 & 8.5 \\
Numbness & 47 & 11.8 \\
Burning & 36 & 9 \\
Radiating & 116 & 29 \\
\hline
\end{tabular}


Figure 2 shows that $44.5 \%$ respondents were suffering from moderate pain, $26.2 \%$ mild, $21.8 \%$ severe, and $7.5 \%$ had intolerable pain.

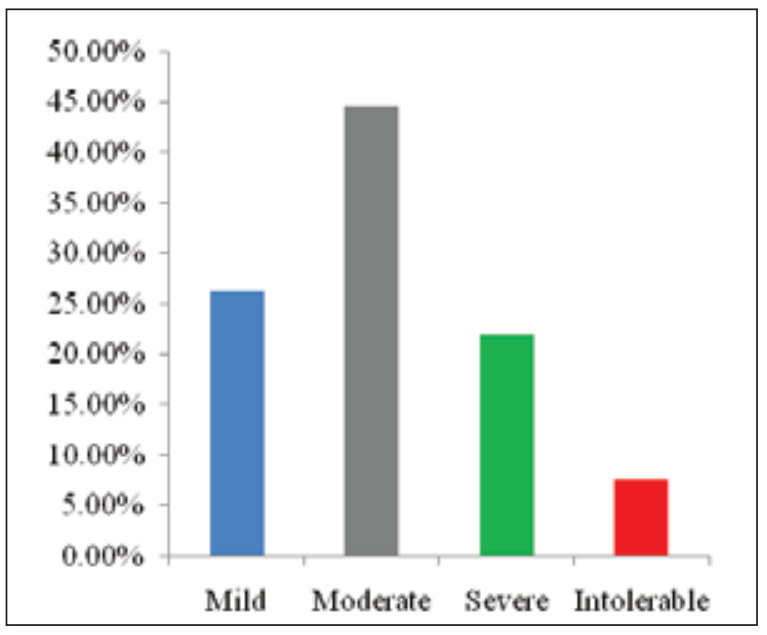

Fig 1: Distribution of respondents by duration of pain

Investigation(s) advised by physicians were done on $85.5 \%$ respondents. Among them 81.8\% respondents had done $\mathrm{x}$-ray, $70.2 \%$ had blood tests of different types, and $22.2 \%$ had MRI. Regarding diagnosis, 29.2\% was diagnosed as muscle spasm, $27.5 \%$ as degenerative diseases, $12.2 \%$ as reduction of lordotic curve, $6.8 \%$ as inter-vertebral disc diseases, $5.8 \%$ as gout and $4 \%$ had no abnormal findings (Table IV).

Table IV: Distribution of respondents by type of investigations and disease diagnosis (Multiple responses)

\begin{tabular}{lcc}
\hline Variables & Frequency & Percentage \\
\hline Investigations & 327 & 81.8 \\
$\quad$ X-ray & 281 & 70.2 \\
$\quad$ Blood test & 89 & 22.2 \\
$\quad$ MRI & & \\
Diagnosis & 16 & 4 \\
No abnormal finding & 117 & 29.2 \\
Muscle spasm & 49 & 12.2 \\
Reduction of lordotic curve & 110 & 27.5 \\
Degenerative diseases & 27 & 6.8 \\
Inter-vertebral disc diseases & 23 & 5.8 \\
Gout & & \\
\hline
\end{tabular}

Table V shows that $83.8 \%$ respondents received medication, $61.2 \%$ physiotherapy, and $3.5 \%$ occupational therapy.

Table V: Distribution of respondents by type of treatment (Multiple responses)

\begin{tabular}{lcc}
\hline Treatment pattern & Frequency & Percentage \\
\hline Medication & 335 & 83.8 \\
Physiotherapy & 245 & 61.2 \\
Occupational therapy & 14 & 3.5 \\
\hline
\end{tabular}

Among physiotherapy, $55.00 \%$ respondents received traction, $91.25 \%$ received exercise, $21.25 \%$ received manipulation, $97.95 \%$ received short wave diathermy, $85.00 \%$ received ultrasound therapy, $33.75 \%$ received infra red ray, and $12.75 \%$ received electrical stimulation (Table VI).

Table VI: Distribution of physiotherapy treatment (Multiple responses)

\begin{tabular}{lcc}
\hline Physiotherapy & Frequency & Percentage \\
\hline Traction & 220 & 55 \\
Exercise & 365 & 91.25 \\
Manipulation & 85 & 21.25 \\
Short wave diathermy & 390 & 97.95 \\
Ultrasound therapy & 340 & 85 \\
Infra red ray & 135 & 33.75 \\
Electrical stimulation & 51 & 12.75 \\
\hline
\end{tabular}

Statistically significant difference was observed between sex and severity of pain and also between educational status and problem persisting time in years (Table VII \& VIII).

Table VII: Distribution and association of respondents between sex and severity of pain $(\mathrm{N}=\mathbf{4 0 0})$

\begin{tabular}{lcccc}
\hline Educational status & \multicolumn{3}{c}{ Problem persist in year } & p-value \\
\hline & $\mathbf{1 - 5}$ years & $\mathbf{6 - 1 0}$ years & $>$ 11 years & \\
Primary & 8 & 2 & 0 & \\
Secondary & 34 & 1 & 0 & \\
Higher secondary & 132 & 3 & 0 & 0.000 \\
Graduate & 138 & 9 & 1 & \\
Post-graduate & 62 & 8 & 2 & \\
\hline p value obtained from Fischer's exact test &
\end{tabular}




\section{Discussion}

This study reveals that common area of musculoskeletal pain were neck in $75.2 \%$, back $48.00 \%$, shoulder $30.8 \%$, upper limb $19 \%$, knee $10.8 \%$, and leg and ankle in $6 \%$ respondents. These findings were similar to that of the study carried out by Movahhed et al. and Oha et al. ${ }^{12,13}$ This study showed that $73.2 \%$ had intermittent pain, $29 \%$ radiating pain, $25.2 \%$ continuous pain, $11.8 \%$ had numbness, and $9 \%$ burning and $8.5 \%$ tingling sensation. These findings were similar to the findings of Silva et al. ${ }^{18}$ Among the respondents $44.5 \%$ were suffering from moderate pain, $26.2 \%$ mild pain, $21.8 \%$ severe pain, and $7.5 \%$ are feeling intolerable pain and these findings were similar to the study carried out by Silva et al. ${ }^{18}$ Study reveals that $55.00 \%$ respondents received traction, $91.25 \%$ received exercise, $21.25 \%$ received manipulation, $97.95 \%$ received short wave diathermy, $85.00 \%$ received ultrasound therapy, $33.75 \%$ received infra red ray, and $12.75 \%$ electrical stimulation. These findings were supported by the findings of Hanada et al. and Shamliyan et al.4,6

There were statistically significant association between sex and severity of pain, educational status and problem persisting time in years $(p=0.019,0.000,<0.05)$. Those findings were similar to the study carried out by Sustová et al. ${ }^{15}$

\section{Conclusion}

The present study found that the common areas of musculoskeletal pain were neck, back, shoulder, upper limb, knee, leg and ankle. Characteristics and intensity of musculoskeletal pain were intermittent, radiating, continuous, numbness, burning, and tingling sensation and moderate to severe and intolerable pain. Respondents had taken different types of physiotherapy services including traction, exercise, manipulation, short wave diathermy, ultrasound therapy, infra red ray, and electrical stimulation.

\section{Disclosure}

All the authors declared no competing interest.

\section{References}

1. Mannion AF, Balagué F, Pellise F and Cedraschi C. Pain Measurement in Patients with Low Back Pain. Nature Clinical Practice Rheumatology. 2007;3(11):610-18.

2. Hills R, Kitchen S. Satisfaction with Outpatient Physiotherapy: Focus Groups to Explore the Views of Patients with Acute and Chronic Musculoskeletal Conditions. Physiotherapy Theory Practice. 2007;23(1):1-20.

3. Roine E, Roine RP, Räsänen P, Vuori I, Sintonen H, Saarto T. Cost-effectiveness of Interventions Based on Physical Exercise in the Treatment of Various Diseases: A Systematic Literature Review. Int J Technol Assess Health Care. 2009;25(4):427-54.

4. Hanada EY. Efficacy of Rehabilitative Therapy in Regional Musculoskeletal Conditions. Best Pract Res Clin Rheumatol. 2003;17(1):151-66.

5. Alexandre NM, Nordin M, Hiebert R, Campello M. Predictors of Compliance with Short-term Treatment among Patients with Back Pain. Rev Panam Salud Publica. 2002;12(2):86-94.

6. Shamliyan TA, Wang S-Y, Olson-Kellogg B, Kane RL. Physical Therapy Interventions for Knee Pain Secondary to Osteoarthritis. Rockville, MD: Agency for Healthcare Research and Quality; 2012 Nov. Comparative Effectiveness Review No. 77. AHRQ Publication No. 12(13)-EHC115-EF.

7. Casserley-Feeney SN, Phelan M, Duffy F, Roush S, Cairns MC, Hurley DA. Patient Satisfaction with Private Physiotherapy for Musculoskeletal Pain. BMC Musculoskelet Disord. 2008;15;9:50.

8. Larsson ME, Kreuter M, Nordholm L. Is Patient Responsibility for Managing Musculoskeletal Disorders Related to Self-reported Better Outcome of Physiotherapy Treatment? Physiother Theory Pract. 2010;26(5):308-17.

9. Magnago TS, de Lima AC, Prochnow A, Ceron MD, Tavares JP, Urbanetto Jde S. Intensity of Musculoskeletal Pain and (in) Ability to Work in Nursing. Rev Lat Am Enfermagem. 2012;20(6):1125-33

10. Ullrich PM, Jensen MP, Loeser JD, Cardenas DD. Pain Intensity, Pain Interference and Characteristics of Spinal Cord Injury. Spinal Cord. 2008;46(6):451-55. 
11. Lopes AD, Costa LO, Saragiotto BT, Yamato TP, Adami F, Verhagen E. Musculoskeletal Pain is Prevalent among Recreational Runners Who are about to Compete: An Observational Study of 1049 Runners. J Physiother. 2011;57(3):179-82.

12. Oha K, Animägi L, Pääsuke M, Coggon D, Merisalu E. Individual and Work-related Risk Factors for Musculoskeletal Pain: A Cross-sectional Study among Estonian Computer Users. BMC Musculoskelet Disord. 2014;15(1):181.

13. Anke $\mathrm{A}$, Damsgård $\mathrm{E}$, Røe $\mathrm{C}$. Life Satisfaction in Subjects with Long-term Musculoskeletal Pain in Relation to Pain Intensity, Pain Distribution and Coping. J Rehabil Med. 2013;45(3):277-85.

14. Sustová Z, Hodacová L, Kapitán M. The Prevalence of Musculoskeletal Disorders among Dentists in the Czech Republic. Acta Medica (Hradec Kralove). 2013;56(4):150-56.
15. Inoue $\mathrm{S}$, Ushida $\mathrm{T}$, Inoue $\mathrm{M}$. Clinical Characteristics and Multidisciplinary Management of Chronic Musculoskeletal Pain. Brain Nerve. 2012;64(11):1287-97.

16. Karttunen NM, Turunen JH, Ahonen RS, Hartikainen SA. Persistence of Nonmalignant Musculoskeletal Chronic Pain among Community-dwelling Older People - A Population Based Longitudinal Study in Finland. Clin J Pain. 2015;31(1):79-85.

17. Silva AG, Alvarelhão J, Queirós A, Rocha NP. Pain Intensity is Associated with Self-reported Disability for Several Domains of Life in a Sample of Patients with Musculoskeletal Pain Aged 50 or More. Disabil Health J. 2013;6(4):369-76.

18. Choi K, Park JH, Cheong HK. Prevalence of Musculoskeletal Symptoms Elated with Activities of Daily Living and Contributing Factors in Korean Adults. J Prev Med Public Health. 2013;46(1):39-49. 